\title{
Novel progresses of chimeric antigen receptor (CAR) T cell therapy in multiple myeloma
}

\author{
Lijuan Ding ${ }^{1}$, Yongxian Hu ${ }^{1,2,3}$, He Huang ${ }^{1,2,3}$ \\ ${ }^{1}$ Bone Marrow Transplantation Center, the First Affiliated Hospital, School of Medicine, Zhejiang University, Hangzhou, China; ${ }^{2}$ Institute of \\ Hematology, Zhejiang University, Hangzhou, China; ${ }^{3}$ Zhejiang Province Engineering Laboratory for Stem Cell and Immunity Therapy, Hangzhou, \\ China \\ Contributions: (I) Conception and design: Y Hu, H Huang; (II) Administrative support: H Huang; (III) Provision of study materials or patients: Y Hu; \\ (IV) Collection and assembly of data: L Ding; (V) Data analysis and interpretation: L Ding, Y Hu; (VI) Manuscript writing: All authors; (VII) Final \\ approval of manuscript: All authors. \\ Correspondence to: He Huang. Bone Marrow Transplantation Center, the First Affiliated Hospital, School of Medicine, Zhejiang University, Hangzhou \\ 310003, China. Email: huanghe@zju.edu.cn.
}

\begin{abstract}
Multiple myeloma ( $M M)$ is a malignant proliferative disease of plasma cells, which leads to suppressed hematopoietic and osteolytic diseases. Despite the use of traditional chemotherapy, hematopoietic stem cell transplantation (HSCT) and targeted drugs, MM still cannot be completely cured. In recent years, chimeric antigen receptor (CAR) $\mathrm{T}$ cells have revolutionized immunotherapy and cancer treatment. The great success of CAR-T cells in leukemia and lymphoma has promoted its development in MM. The primary requisite for developing clinically effective CAR-T cells suitable for MM is to identify the appropriate targets. In early clinical trials, CAR-T cells targeting B-cell maturation antigen (BCMA) have shown significant anti-MM activity. Currently popular targets in clinical research and preclinical research include CD138, CD38, CS1, CD19, $\kappa$ light chain, CD56, CD44v6, Lewis Y, NY-ESO-1, CD229, etc. Common toxicities such as cytokine release syndrome (CRS) and neurotoxicity also occur but controllable. MM cells are mainly localized in bone marrow, therefore, the bone marrow microenvironment has a significant effect on the therapeutic effect of CAR-T cells. Targeting both MM cells and the bone marrow microenvironment is currently the most promising treatment. In this review, we provide a comprehensive overview of CAR-T cell therapy in MM, as well as outline potential targets and methods that can overcome local immunosuppression and improve the efficacy of CAR-T cells.
\end{abstract}

Keywords: Chimeric antigen receptor T cells (CAR-T cells); multiple myeloma (MM); target; B-cell maturation antigen (BCMA); microenvironment

Received: 27 June 2020; Accepted: 11 December 2020; Published: 15 January 2021.

doi: $10.21037 /$ sci-2020-029

View this article at: http://dx.doi.org/10.21037/sci-2020-029

\section{Introduction}

Multiple myeloma (MM) is the second largest malignant tumor in the hematopoietic system, accounting for about $10 \%$ of hematopoietic malignancies. It is characterized by massive proliferation of monoclonal plasma cells, which can eventually cause renal failure, anemia, bone destruction and repeated infections related to immunosuppression (1). Current treatments include supportive treatment, high- dose chemotherapy, and autologous hematopoietic stem cell transplantation (HSCT). With the advent of immune modulators such as thalidomide, lenalidomide, and proteasome inhibitor bortezomib in the past ten years, the treatment of MM has made significant progress, and the median survival time has been extended to about 6 years (2). However, after initial effective chemotherapy, most patients still face fatal recurrence, which remains a major challenge in the treatment of MM. 


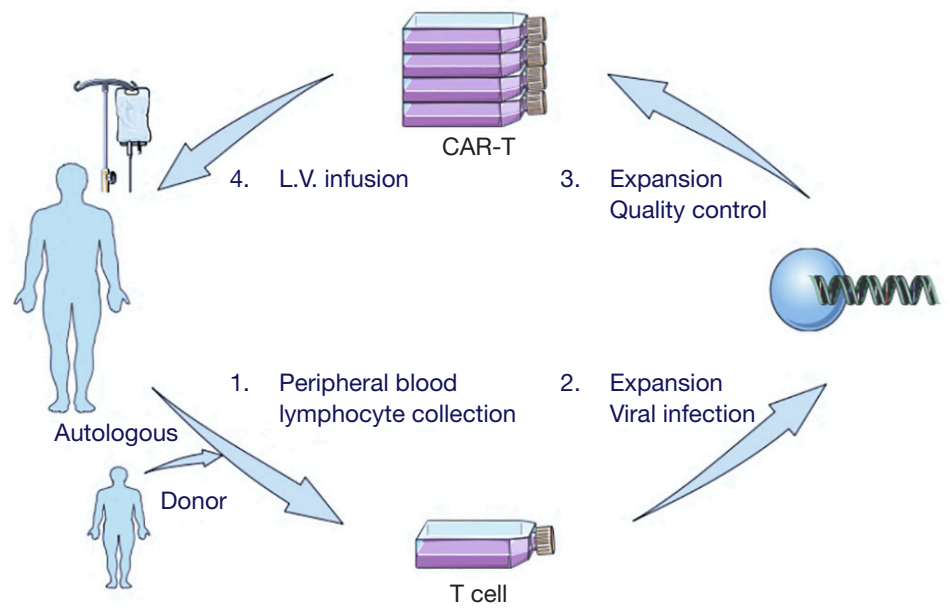

Figure 1 Process of CAR-T treatment. (I) Collect lymphocyte from patients' peripheral blood and isolate T cells; (II) infect T cells with CAR lentivirus; (III) before infusion, expand CAR-T cells and control the quality; (IV) infuse CAR-T cells to patients according to weight. CAR, chimeric antigen receptor.

The Mayo Center analyzed 286 MM patients resistant to bortezomib and immunomodulators or relapsed after these therapies. The median event-free survival (EFS) period was only 5 months, and the median overall survival (OS) period was 9 months. These results suggest that the prognosis for patients resistant to bortezomib and primary/ secondary immunomodulators is extremely poor (3). Other new drugs include 5 -type phosphodiesterase inhibitors that target the bone marrow microenvironment, PD-1/PD-L1 signaling pathway inhibitors, histone deacetylase inhibitors, monoclonal antibody CD38 mAb, B-cell activating factor mAb, etc. $(4,5)$. The emergence of the above drugs has improved the efficacy and prognosis of MM patients, but most of them exert antitumor effects by changing the growth environment of tumor cells, without specifically affect all stages of tumor cells. Therefore, it is necessary to address more effective, more specific, and less toxic therapies to fully realize the anti-myeloma potential of the immune system.

\section{Chimeric antigen receptor (CAR)-T therapy in MM}

CAR-T cells have been successfully used in the treatment of B cell malignancies as a new method of tumor immunotherapy. $\mathrm{T}$ cells are infected with lentivirus in vitro to manufacture CAR-T cells, which can specifically recognize and kill target cells. The CAR construct is mainly composed of two parts, the extracellular antigen recognition part and the intracellular activation part (6). The signal is formed by the $\zeta$ chain of CD3 complex and costimulatory molecule, thereby promoting $\mathrm{T}$ cell proliferation, releasing cytokines, and resisting cell apoptosis. The treatment process is as follows: (I) $\mathrm{T}$ cell extraction from peripheral blood of patient or donor; (II) $\mathrm{T}$ cell modification with CAR constructs in vitro through lentivirus infection; (III) mass expansion of CAR-T cells and strict quality control; (IV) CAR-T cell infusion after lymphocyte depletion chemotherapy (Figure 1).

Due to our limited knowledge about CAR-T cells, the timing of employing CAR-T therapy is mostly the case where patients have repeatedly failed to traditional treatments. In fact, this situation should be changed. For refractory patients, doctors generally increase treatment intensity, or change treatment plan. This will lead to two results: (I) remission again, combined with severe damage to patients' normal organs and serious complications; the probability of remission is actually not great. (II) Ineffective, also accompanied by serious complications and impaired organ function. Therefore, for patients with refractory MM, CAR-T cell therapy should be adopted as soon as possible after conventional induction therapy is ineffective.

So far, the clinical trials of CAR-T cells in MM have exceeded 120 (Table 1). The trials are mainly carried out in US and China. The data is continuously updated on ClinicalTrials.gov, indicating that CAR-T cells have great 
potential in MM. (Data updated to June 1, 2020).

\section{Target selection}

The key factor that determines the success of CAR-T cell therapy is the identification of specific and ideal target antigens. To prevent tumor cells from escaping, all tumor

Table 1 World distribution of clinical trials of CAR-T cells in MM

\begin{tabular}{lc}
\hline Country & The number of registered clinical trials \\
\hline USA & 55 \\
China & 45 \\
Italy & 8 \\
Germany & 6 \\
Spain & 6 \\
France & 5 \\
Japan & 4 \\
Canada & 4 \\
Australia & 3 \\
\hline
\end{tabular}

CAR, chimeric antigen receptor; MM, multiple myeloma. cells must express the target antigen, or the target antigen is essential for the maintenance of tumor gene phenotype. At the same time, the target antigen cannot be expressed on cells of important organs or tissues (such as hematopoietic stem cells) in case of lethal danger. MM cells express many molecules, including CD138, CD56, CD38, CS1, BCMA, CD19, CD70, CD44v6, immunoglobulin light chain, etc. (Table 2). Considering the above requirements, not all of these molecules are suitable as target antigens for CAR-T cells.

\section{B-cell maturation antigen (BCMA)}

Recently, researchers have been focusing on BCMA as a target for CAR-T cells in MM. BCMA, a member of tumor necrosis family receptor (TNFR), is expressed on terminally differentiated $\mathrm{B}$ cells with high restrictions. BCMA antibodies have killing effects on both MM cell lines and primary MM cells (23). The number of B cells in BCMAdeficient mice is normal, but B cell function is inhibited (24). The above results suggest that BCMA is suitable as a target for $M M$ treatment, and will not cause a significant impact on the function of normal B cells. Clinical trials using

Table 2 Expression of potential targets for CAR-T cell therapy in MM

\begin{tabular}{|c|c|c|c|}
\hline Target & Expression on MM cells (\% of cases) & Expression on normal cells & References \\
\hline \multicolumn{4}{|c|}{ Targets of clinical trials } \\
\hline CD138 & $90-100 \%$ & Plasma cells & $(7,8)$ \\
\hline CS1 & $95 \%$ & Plasma cells, T cells, NK cells, NK-T cells & $(9,10)$ \\
\hline CD38 & $80-100 \%$ & $\begin{array}{l}\text { Monocytes, early B cells, activated T cells, } \\
\text { NK cells }\end{array}$ & $(11,12)$ \\
\hline BCMA & $60-100 \%$ & Plasma cells, mature B cells & $(13,14)$ \\
\hline CD19 & $\begin{array}{l}\text { Low expression, mostly on disease } \\
\text { propagating cells }\end{array}$ & B cells & $(15)$ \\
\hline$\kappa$ light chain & $\begin{array}{l}\text { Expression on } \kappa \text {-restricted disease } \\
\text { propagating cells }\end{array}$ & Mature B cells & $(16)$ \\
\hline \multicolumn{4}{|c|}{ Targets of preclinical experiments } \\
\hline CD56 & $60-80 \%$ & NK cells, NK-T cells & $(17,18)$ \\
\hline CD44v6 & $43 \%$ (in advanced stage) & Monocytes, activated T cells & $(19)$ \\
\hline Lewis $\mathrm{Y}$ & $52 \%$ & Plasma cells & (20) \\
\hline NY-ESO-1 & $60 \%$ & & $(21)$ \\
\hline CD229 & $100 \%$ & T cells, B cells, plasma cells & (22) \\
\hline
\end{tabular}

CAR, chimeric antigen receptor; MM, multiple myeloma. 


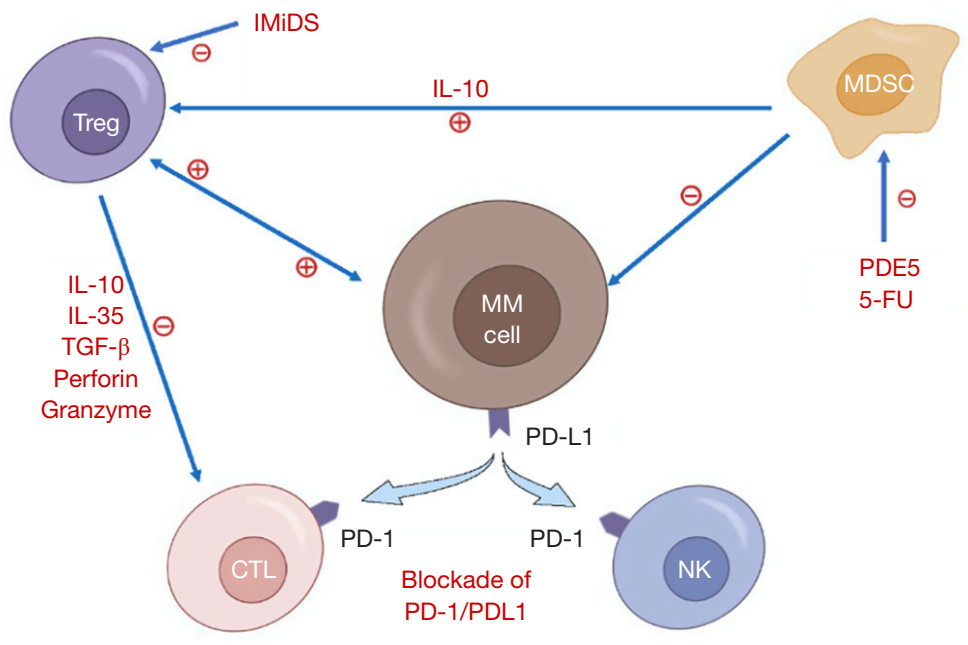

Figure 2 Models of bone marrow microenvironment in MM. The blue arrows indicate the pathways which can be targeted in order to achieve an immunosuppressive effect. The red signs indicate the effect of promoting or inhibiting. MM, multiple myeloma; IMiDs, immunomodulatory drugs; MDSC, myeloid-derived suppressor cells; Treg, T regulatory cells; DC, dendritic cells; CTL, cytotoxic T lymphocyte; NK, natural killer cell; Th1, type 1 helper T cells; PDE5, phosphodiesterase type 5; 5-FU, 5-fluorouracil; TGF- $\beta$, transforming growth factor beta; IL-35, interleukin-35; IL-10, interleukin-10.

BCMA CAR-T cells have exceeded 60 items by June 1, 2020. Ali et al. demonstrated for the first time that BCMA CAR-T cells can recognize and kill human MM cell lines as well as primary MM cells (14). Ali et al. published the results of the first clinical trial with second-generation BCMA CAR-T cells, in which 12 patients received four dose gradient treatment $\left(0.3,1,3,9 \times 10^{6} / \mathrm{kg}\right.$ CAR-T cells). The number of BCMA CAR-T cells in the peripheral blood changed in a dose-dependent manner. The best response was strict complete response (CR) $(\mathrm{n}=1)$, which occurred in the highest dose group. Other responses included very good partial response (VGPR) $(\mathrm{n}=2)$, partial response $(\mathrm{PR})(\mathrm{n}=1)$ and stable disease $(\mathrm{SD})(\mathrm{n}=8)$. Berdeja et al. reported a multi-center study sponsored by Bluebird Bio at the 2017 ASH conference (25). The study used bb2121, which included a murine anti-BCMA scFv and a 4-1BB costimulatory domain. Four dose levels (50, 150, 450, and $800 \times 10^{6}$ CAR-T cells) were explored in 21 patients with relapsed/refractory multiple myeloma (RRMM), in which 17 patients (81\%) responded. Ten patients achieved CR, with a median follow-up time of 40 weeks. Only 4 patients responded subsequently progressed and did not reach median progression-free survival. The continuous response time of 5 patients exceeded 1 year.

Currently, ongoing researches show that BCMA CAR-T cells have considerable potential for anti-MM efficacy and do not cause uncontrollable cytokine release syndrome (CRS) or unexpected toxicity. However, further clinical data are still needed to determine the durability of the treatment response, as well as the optimal CAR structure and cell product composition to maximize the benefit for MM patients.

\section{CD19}

CD19 CAR-T cells have been approved by the US Food and Drug Administration (FDA) and can be used to treat ALL and DLBCL. Most MM cells are fully differentiated plasma cells without CD19 expression. However, a small proportion of MM cells remain in the early stage of differentiation, accompanied by positive expression of CD19, and are related with drug resistance and the ability to promote disease progression $(26,27)$. In fact, CD19 expression seems to be more common in MM cells than generally thought and is associated with poor survival $(15,28)$. Garfall et al. conducted a prospective study to explore the use of CD19 CAR-T cells after high-dose melphalan and salvage autologous HSCT in RRMM. Ten patients who relapsed 1 year after first autologous HSCT received second autologous HSCT, and were infused with (1 to 5$) \times 10^{7}$ CD19 CAR-T cells [12-14] days after transplantation. The team published a case report in 
which the patient achieved MRD-negative CR that lasted 12 months (15). The team is also conducting another study, in which CD19 CAR-T cells are infused during the maintenance period of approximately 60 days after first autologous HSCT.

CD19 CAR-T cells have also been used in combination with BCMA CAR-T cells. The Affiliated Hospital of Xuzhou Medical University in China conducted a singlecenter, single-arm phase II trial, in which 21 patients with RRMM received CD19 CAR-T cells and BCMA CAR-T cells (29). At a median follow-up time of 179 days, 20 patients (95\%) had overall remission, including 9 (43\%) strict CRs, 3 (14\%) CRs, 5 (24\%) VGPRs, and 3 (14\%) PRs. The most common adverse reaction was CRS (19/21, 90\%). The results confirmed that combination of CD19 and BCMA CAR-T cells is feasible in RRMM patients, and the initial activity observed is worthy of further study in randomized trials.

\section{CD38}

CD38 molecule is a single-chain type II transmembrane glycoprotein, which is highly expressed on the surface of myeloma cells, including MGUS, MM, extramedullary plasmacytoma and plasma cell leukemia (30). Therefore, CD38 targeted therapy is a potential treatment for MM. Anti-CD38 monoclonal antibodies such as daratumumab have a certain therapeutic effect on $\operatorname{RRMM}(5,31)$. Compared with anti-CD38 monoclonal antibodies, CD38 CAR-T cells can fully improve the specificity of CD38targeting antibodies, and have a durable and efficient response reaction. However, the expression distribution of CD38 is quite extensive, including skeletal muscle, cardiac muscle, airway smooth muscle and uterine smooth muscle. Therefore, reducing the occurrence of adverse reactions is particularly important. Recently, Drent et al. simulated the human body environment in MM mouse model, and tried to introduce the caspase-9 (iCasp9) suicide gene into CD38 CAR-T cells (11). The results suggest that CD38 CAR-T cells have great potential for the treatment of CD38+ malignant tumors and are feasible to control adverse reactions through iCasp9 suicide gene, which are of great significance for future clinical trials of CD38 CAR-T cells.

\section{CD138}

CD138, also known as syndecan-1, is a heparan sulfate proteoglycan expressed on the surface of plasma cells and part of epithelial cells at various stages of differentiation. CD138 as a basic diagnostic indicator of MM is closely related to the tumor growth and disease progression of MM. CD138 is expressed on the surface of normal and malignant plasma cells and is the main diagnostic marker of MM (32). Preclinical studies using CD138 CAR-NK cells have shown effective anti-MM activity both in vitro and in vivo (33). Therefore, CD138 is a very attractive target for MM. One study reported the results of CD138 CAR-T cells in 5 RRMM patients, with each patient infused with an average of $0.76 \times 10^{7}$ total cells $/ \mathrm{kg}$. The cells expanded in all patients but no objective response was observed. Four patients remained SD for 3 to 6 months. Although targeting CD138 in the treatment of MM has strong appeal, CD138 CAR-T cells have been found to be significant in skin and/or mucosal toxicity in pre-clinical studies of MM treatment (34). In the future, relevant strategies may be needed to avoid targeted toxicity while maintaining potential anti-tumor effects.

\section{CS1}

CS1, also known as signaling lymphocytic activation molecule 7 (SLAMF7), is a member of the SLAM transmembrane receptor family. CS1 is poorly expressed on NK cells, T cells and B cells, but is widely expressed on the surface of myeloma cells, including relapsed MM patients (10). Chu et al. Constructed CS1 CAR-T cells and tested the killing function using orthotopic MM xenograft mouse model. The results proved that CS1 CAR-T cells can effectively eliminate human myeloma cells and prolong the survival time of mice (35). At present, several CAR-T cells targeting CS1 have been successfully developed. Preclinical studies show that NK cells can also be genetically edited to be effective against MM. The advantage of CAR-NK cells is that they can use allogenic NK cells to reduce the incidence of graft-versus-host disease (GVHD). However, CARNK cells may trigger a series of cytotoxic reactions due to the mismatch between the killer cell immunoglobulin-like receptor (KIR) on their surface and non-target cells (36).

\section{Immunoglobulin kappa light chain}

B cells and plasma cells can express kappa or lambda chains on their surfaces. The defect of kappa light chain does not lead to a significant down-regulation of humoral immunity 
and up-regulation of susceptibility to pathogens (37). In MM patients, the positive detection rate of monoclonal kappa light chain antigen on the surface of MM cells expressing only kappa light chain was $70 \%$, while no monoclonal lambda light chain antigen was detected on the surface of MM cells expressing lambda light chain (38). The above research results suggest that kappa light chain antigen can be used as a target for MM immunotherapy. Researchers at Baylor College of Medicine in the United States designed the first- and second-generation CAR-T cells targeting kappa light chain, and compared the tumoricidal effects of the two generations on kappa light chain MM mice. The results show that the first and second generations can specifically kill kappa-positive MM cells and promote the massive release of cytokines such as IL-2, IFN- $\gamma$, and TNF- $\alpha$. The second-generation CAR-T cells reduce tumor signals more significantly (39).

\section{CD44v6}

CD44 is selectively and highly expressed in hematopoietic tumors and epithelial tumors, and is considered to be one of the markers of tumor stem cells. The expression of CD44 isoform $6(\mathrm{CD} 44 \mathrm{v} 6)$ is relatively tumor-limited and has been reported to be associated with poor prognosis in MM (19). Pre-clinical studies have demonstrated that CD44v6 CAR-T cells exhibit strong anti-tumor activity against $M M$ and have transiently reversible monocyte reduction in mouse models. This side effect may be beneficial because monocytes have been proved to be the main cause of CRS (40). The safety switch in the form of suicide gene has greatly reduced the risk of toxicity (19).

\section{CD56}

CD56 is a surface glycoprotein that mediates cell-cell and cell-matrix interactions. CD56 is strongly expressed by malignant plasma cells in $70 \%$ of myeloma patients and represents a potential immunotherapy target. CD56 is also expressed at lower levels on normal tissue types (including neuronal cells, NK cells, and parts of activated T cells) (41). In vitro cytotoxicity tests show that CD56 CAR-T cells have a significant lysis effect on myeloma cell lines, and can effectively eradicate tumor cells in MM mice at a dose of $5 \times 10^{6}$ (18). Therefore, CD56 CAR-T therapy is a prospective immunotherapy and should be considered for RRMM patients.

\section{Lewis $Y$}

Lewis $\mathrm{Y}$ antigen is a tetrasaccharide structure that binds to the oligosaccharide chain of type II blood group, and is significantly expressed in epithelial tumors (including breast cancer, colon cancer, ovarian cancer, lung cancer, etc.). A second-generation CAR test with Lewis $\mathrm{Y}$ as the target antigen showed that it had a good tumor killing effect on nude mice transplanted with ovarian cancer subcutaneously (42). Peinert et al. found that Lewis $\mathrm{Y}$ antigen was expressed in $52 \%$ of MM patients, and proved the efficacy of Lewis $\mathrm{Y}$ CAR-T cells in vitro and in vivo in NOD/SCID mouse models (20). Neeson et al. found that Lewis Y CAR-T cells can not only express perforin and granzyme to exert cytotoxicity, but also be polarized to the phenotype of central memory $\mathrm{T}$ cells, indicating that Lewis Y CAR-T cells have the potential to proliferate continuously (43). At present, CAR-T cells targeting Lewis $\mathrm{Y}$ antigen have been clinically tested in the treatment of MM.

\section{NY-ESO-1}

Many malignant tumors highly express NY-ESO-1, including melanoma, prostate cancer, lung cancer, breast cancer, etc., while normal tissues do not express or lowly express NY-ESO-1. About $60 \%$ of MM patients express NY-ESO-1, and the expression level is higher in patients with recytogenetic abnormalities and relapses, suggesting that NY-ESO-1 is closely related to the progression of MM disease (21). Preclinical trials have confirmed the targeted therapeutic effect of NY-ESO-1 CAR-T cells, the ability to secrete cytokines and induce immune memory (44).

\section{CD229}

CD229 is a molecule that is generally expressed on the surface of myeloma cells and myeloma stem cells. It is one of the SLAM family receptors, also known as SLAM3 (45). The high expression on MM cells suggests that CD229 has great potential as a target for CAR-T immunotherapy. Radhakrishnan et al. Designed a CAR construct targeting CD229. Studies have shown that this newly designed CD229 CAR-T cell has high activity against MM cells, memory $B$ cells and MM stem cells in vitro and in vivo (22). The elimination of MM stem cells improves the durability of the treatment response. CD229 CAR-T cells will be pushed into clinical trials after safety verification. 


\section{CAR-T toxicities}

The toxicities of CAR-T cells in MM are similar to those reported for CD19 CAR-T cells in ALL and lymphoma, with most cases being CRS and neurotoxicity $(46,47)$. CAR-T cells recognize tumor antigens and initial the signaling cascade in vivo, which causes massive cytokine release, and activates other inflammatory cells at the same time (48). Explosive release of cytokines can trigger systemic inflammatory reactions, resulting in organ and vascular endothelium damage, and causing serious complications such as heart failure, pulmonary edema, respiratory distress, and microvascular leakage. Grade 3-4 CRS is an indication for the use of glucocorticoids or tocilizumab (49). Neurotoxicity can manifest as central and peripheral nervous system symptoms such as headache, vomiting, vision change, tremor, aphasia, lethargy, hemiplegia, epilepsy, and myoclonus. Neurotoxicity is usually selflimited and requires only supportive treatment. Small doses of glucocorticoids can be used in severe cases.

\section{Improving the efficacy of CAR-T therapy in MM}

Although CAR-T cell therapy has significantly improved the clinical outcome of $M M$, it fails to completely cure MM because of the huge clonal heterogeneity and immunosuppressive bone marrow microenvironment. MM cells grow and expand almost completely in the bone marrow, therefore, the microenvironment and its various components are particularly important in supporting the growth and survival of MM cells. Different types of immune cells in the bone marrow microenvironment inhibit $\mathrm{T}$ cell function (Figure 2). In particular, T regulatory cells (Tregs) use multiple mechanisms to suppress effector $\mathrm{T}$ cells, such as secreting inhibitory cytokines (TGF- $\beta$, IL-10 and IL-35) and cytolytic granules (granzyme, perforin) (50). According to reports, the concentration of TGF- $\beta$ and IL-10 in the bone marrow of MM patients is very high, indicating an immunosuppressive microenvironment (51). Cyclophosphamide can reduce the number and function of Tregs, enhancing anti-tumor immunity (52). Immunomodulatory drugs (IMiDs) (i.e., lenalidomide and pomalidomide) inhibit the expansion and function of Tregs by reducing FOXP3 mRNA expression (53). It is essential to understand how bone marrow microenvironment regulates the development and function of Tregs in order to develop more specific and effective therapies.

The number of bone marrow-derived suppressor cells
(MDSCs) in the peripheral blood and bone marrow of MM increases. MDSCs can secrete cytokines (such as IL10) to induce the development of Tregs in tumor-bearing mouse models, resulting in a more suppression immune environment (54). Görgün et al. reported the direct effect of MDSCs on MM growth in vitro (55). Phosphodiesterase 5 (PDE5) inhibitors can reduce the inhibitory activity of MDSCs by inhibiting the degradation of cyclic guanosine monophosphate (cGMP), and reduce the expression of arginase 1 (ARG1) and nitric oxide synthase 2 (NOS2) in tumor models $(56,57)$. The pyridine analog 5 -fluorouracil (5-FU) has an effect on MDSCs depletion and restores $\mathrm{T}$ cell-specific immune responses (58). Considering that new anti-MM drugs (such as bortezomib) target not only MM cells, but also the bone marrow microenvironment (59), the effects of these drugs on MDSCs were studied. However, none of these drugs can change the inhibitory function of MDSCs (55). This shows that new strategies are needed for MDSCs.

Co-suppressor molecule PD-1 is overexpressed on T cells of MM patients, and its ligand PD-L1 is expressed on MM cells. The interaction of PD-1/PD-L1 may play an important role in the local immunosuppression of MM (4). Blockade of PD-1 signaling prevents or reduces $\mathrm{T}$ cell tolerance and restore tumor immunity (60). The application of anti-PD-1 antibodies has been found to accumulate CD8+ T cells with memory precursor phenotype in breast cancer mice, which enhanced the killing ability of $\mathrm{T}$ cells (61).

The above findings indicate that in addition to selecting suitable targets, CAR-T cell therapy in $M M$ also requires a joint strategy targeting the bone marrow microenvironment.

\section{Conclusions and perspectives}

CAR-T cell therapy provides us with a very effective tool and becomes part of our arsenal of cancer treatments. Although the response rate of CAR-T cell therapy in RRMM is exciting, the efficacy is difficult to prolong. Most patients will relapse within a few months with disease progression. Therefore, we must find ways to adapt this new type of immunotherapy to the biological characteristics of MM to improve its efficacy. We believe that two methods are particularly promising: one is to identify more suitable targets, and the other is to reverse local immunosuppression in the microenvironment. In addition, whether CAR-T cell therapy can be used in earlier disease stages and its combined application with other medications are also worth further exploration. CAR-T cell therapy is still in its infancy 
in $M M$ and requires a large number of basic and clinical studies. We hope that CAR-T cell technology will bring revolutionary changes to the treatment of MM patients in the near future.

\section{Acknowledgments}

Funding: This work was supported by the grants from Key Project of Science and Technology Department of Zhejiang Province (2019C03016).

\section{Footnote}

Conflicts of Interest: All authors have completed the ICMJE uniform disclosure form (available at http://dx.doi. org/10.21037/sci-2020-029). Dr. HH serves as an unpaid associate editor of Stem Cell Investigation. The other authors have no conflicts of interest to declare.

Ethical Statement: The authors are accountable for all aspects of the work in ensuring that questions related to the accuracy or integrity of any part of the work are appropriately investigated and resolved.

Open Access Statement: This is an Open Access article distributed in accordance with the Creative Commons Attribution-NonCommercial-NoDerivs 4.0 International License (CC BY-NC-ND 4.0), which permits the noncommercial replication and distribution of the article with the strict proviso that no changes or edits are made and the original work is properly cited (including links to both the formal publication through the relevant DOI and the license). See: https://creativecommons.org/licenses/by-nc-nd/4.0/.

\section{References}

1. Palumbo A, Anderson K. Multiple myeloma. N Engl J Med 2011;364:1046-60.

2. Kumar SK, Dispenzieri A, Lacy MQ, et al. Continued improvement in survival in multiple myeloma: changes in early mortality and outcomes in older patients. Leukemia 2014;28:1122-8.

3. Kumar SK, Lee JH, Lahuerta JJ, et al. Risk of progression and survival in multiple myeloma relapsing after therapy with IMiDs and bortezomib: a multicenter international myeloma working group study. Leukemia 2012;26:149-57.

4. Atanackovic D, Luetkens T, Kroger N. Coinhibitory molecule PD-1 as a potential target for the immunotherapy of multiple myeloma. Leukemia 2014:28:993-1000.

5. Plesner T, Arkenau HT, Gimsing P, et al. Phase 1/2 study of daratumumab, lenalidomide, and dexamethasone for relapsed multiple myeloma. Blood 2016;128:1821-8.

6. Davila ML, Sadelain M. Biology and clinical application of CAR T cells for B cell malignancies. Int J Hematol 2016;104:6-17.

7. Guo B, Chen M, Han Q, et al. CD138-directed adoptive immunotherapy of chimeric antigen receptor (CAR)modified $\mathrm{T}$ cells for multiple myeloma. J Cell Immunother 2016;2:28-35.

8. Kawano Y, Fujiwara S, Wada N, et al. Multiple myeloma cells expressing low levels of CD138 have an immature phenotype and reduced sensitivity to lenalidomide. Int J Oncol 2012;41:876-84.

9. Boles KS, Stepp SE, Bennett M, et al. 2B4 (CD244) and CS1: novel members of the CD2 subset of the immunoglobulin superfamily molecules expressed on natural killer cells and other leukocytes. Immunol Rev 2001;181:234-49.

10. Hsi ED, Steinle R, Balasa B, et al. CS1, a potential new therapeutic antibody target for the treatment of multiple myeloma. Clin Cancer Res 2008;14:2775-84.

11. Drent E, Groen RW, Noort WA, et al. Pre-clinical evaluation of $\mathrm{CD} 38$ chimeric antigen receptor engineered T cells for the treatment of multiple myeloma. Haematologica 2016;101:616-25.

12. Tembhare P, Yuan C, Korde N, et al. Antigenic drift in relapsed extramedullary multiple myeloma: plasma cells without CD38 expression. Leuk Lymphoma 2012;53:721-4.

13. Novak AJ, Darce JR, Arendt BK, et al. Expression of BCMA, TACI, and BAFF-R in multiple myeloma: a mechanism for growth and survival. Blood 2004;103:689-94.

14. Ali SA, Shi V, Maric I, et al. T cells expressing an anti-Bcell maturation antigen chimeric antigen receptor cause remissions of multiple myeloma. Blood 2016;128:1688-700.

15. Garfall AL, Maus MV, Hwang WT, et al. Chimeric antigen receptor T cells against $\mathrm{CD} 19$ for multiple myeloma. $\mathrm{N}$ Engl J Med 2015;373:1040-7.

16. Ramos CA, Savoldo B, Torrano V, et al. Clinical responses with $\mathrm{T}$ lymphocytes targeting malignancy-associated $\mathrm{\kappa}$ light chains. J Clin Invest 2016;126:2588-96.

17. Rawstron A, Barrans S, Blythe D, et al. Distribution of myeloma plasma cells in peripheral blood and bone marrow correlates with CD56 expression. Br J Haematol 1999;104:138-43.

18. Benjamin R, Condomines M, Gunset G, et al. CD56 
targeted chimeric antigen receptors for immunotherapy of multiple myeloma. Cancer Res 2012;72:abstr 3499.

19. Casucci M, Nicolis di Robilant B, Falcone L, et al. CD44v6-targeted T cells mediate potent antitumor effects against acute myeloid leukemia and multiple myeloma. Blood 2013;122:3461-72.

20. Peinert S, Prince HM, Guru PM, et al. Gene-modified T cells as immunotherapy for multiple myeloma and acute myeloid leukemia expressing the Lewis Y antigen. Gene Ther 2010;17:678-86.

21. van Rhee F, Szmania SM, Zhan F, et al. NY-ESO-1 is highly expressed in poor-prognosis multiple myeloma and induces spontaneous humoral and cellular immune responses. Blood 2005;105:3939-44.

22. Radhakrishnan SV, Luetkens T, Scherer SD, et al. CD229 CAR T cells eliminate multiple myeloma and tumor propagating cells without fratricide. Nat Commun 2020;11:798.

23. Ryan MC, Hering M, Peckham D, et al. Antibody targeting of B-cell maturation antigen on malignant plasma cells. Mol Cancer Ther 2007;6:3009-18.

24. Xu S, Lam KP. B-cell maturation protein, which binds the tumor necrosis factor family members BAFF and APRIL, is dispensable for humoral immune responses. Mol Cell Biol 2001;21:4067-74.

25. Berdeja JG, Lin Y, Raje N, et al. Clinical remissions and limited toxicity in a first-in-human multicenter study of bb2121, a novel anti-BCMA CAR T cell therapy for relapsed/refractory multiple myeloma. Eur J Cancer 2016;1:S5.

26. Matsui W, Wang Q, Barber JP, et al. Clonogenic multiple myeloma progenitors, stem cell properties, and drug resistance. Cancer Res 2008;68:190-7.

27. Paiva B, Puig N, Cedena MT, et al. Differentiation stage of myeloma plasma cells: biological and clinical significance. Leukemia 2017;31:382-92.

28. Mateo G, Montalban MA, Vidriales MB, et al. Prognostic value of immunophenotyping in multiple myeloma: a study by the PETHEMA/GEM cooperative study groups on patients uniformly treated with high-dose therapy. J Clin Oncol 2008;26:2737-44.

29. Yan Z, Cao J, Cheng H, et al. A combination of humanised anti-CD19 and anti-BCMA CAR T cells in patients with relapsed or refractory multiple myeloma: a single-arm, phase 2 trial. Lancet Haematol 2019;6:e521-9.

30. Harada H, Kawano MM, Huang N, et al. Phenotypic difference of normal plasma cells from mature myeloma cells. Blood 1993;81:2658-63.
31. Lonial S, Weiss BM, Usmani SZ, et al. Daratumumab monotherapy in patients with treatment-refractory multiple myeloma (SIRIUS): an open-label, randomised, phase 2 trial. Lancet 2016;387:1551-60.

32. Nageshwari B, Merugu R. Effect of levamisole on expression of CD138 and interleukin-6 in human multiple myeloma cell lines. Indian J Cancer 2017;54:566-71.

33. Jiang $H$, Zhang $W$, Shang $P$, et al. Transfection of chimeric anti-CD138 gene enhances natural killer cell activation and killing of multiple myeloma cells. Mol Oncol 2014;8:297-310.

34. Sun C, Mahendravada A, Ballard B, et al. Safety and efficacy of targeting CD138 with a chimeric antigen receptor for the treatment of multiple myeloma. Oncotarget 2019;10:2369-83.

35. Chu J, He S, Deng Y, et al. Genetic modification of T cells redirected toward CS1 enhances eradication of myeloma cells. Clin Cancer Res 2014;20:3989-4000.

36. Chu J, Deng Y, Benson DM, et al. CS1-specific chimeric antigen receptor (CAR)-engineered natural killer cells enhance in vitro and in vivo antitumor activity against human multiple myeloma. Leukemia 2014;28:917-27.

37. Pricop L, Hatakeyama A, Isobe H, et al. Analysis of lambda repertoire in kappa-deficient mice. Clin Immunol Immunopathol 1995;76:S179-87.

38. Hutchinson AT, Jones DR, Raison RL. Preclinical and clinical development of an anti-kappa free light chain $\mathrm{mAb}$ for multiple myeloma. Mol Immunol 2015;67:89-94.

39. Vera J, Savoldo B, Vigouroux S, et al. T lymphocytes redirected against the kappa light chain of human immunoglobulin efficiently kill mature B lymphocytederived malignant cells. Blood 2006;108:3890-7.

40. Norelli M, Camisa B, Barbiera G, et al. Monocyte-derived IL-1 and IL-6 are differentially required for cytokinerelease syndrome and neurotoxicity due to CAR T cells. Nat Med 2018;24:739-48.

41. Skerget M, Skopec B, Zadnik V, et al. CD56 expression is an important prognostic factor in multiple myeloma even with bortezomib induction. Acta Haematol 2018;139:228-34.

42. Westwood JA, Smyth MJ, Teng MW, et al. Adoptive transfer of $T$ cells modified with a humanized chimeric receptor gene inhibits growth of Lewis-Yexpressing tumors in mice. Proc Natl Acad Sci U S A 2005;102:19051-6.

43. Neeson P, Shin A, Tainton KM, et al. Ex vivo culture of chimeric antigen receptor $\mathrm{T}$ cells generates functional CD8+ T cells with effector and central memory-like 
phenotype. Gene Ther 2010;17:1105-16.

44. Schuberth PC, Jakka G, Jensen SM, et al. Effector memory and central memory NY-ESO-1-specific redirected $\mathrm{T}$ cells for treatment of multiple myeloma. Gene Ther 2013;20:386-95.

45. Yousef S, Kovacsovics-Bankowski M, Salama ME, et al. CD229 is expressed on the surface of plasma cells carrying an aberrant phenotype and chemotherapyresistant precursor cells in multiple myeloma. Hum Vaccin Immunother 2015;11:1606-11.

46. Maude SL, Laetsch TW, Buechner J, et al. Tisagenlecleucel in children and young adults with B-cell lymphoblastic leukemia. N Engl J Med 2018;378:439-48.

47. Neelapu SS, Tummala S, Kebriaei P, et al. Chimeric antigen receptor T cell therapy -assessment and management of toxicities. Nat Rev Clin Oncol 2018;15:47-62.

48. Kochenderfer JN, Dudley ME, Feldman SA, et al. B-cell depletion and remissions of malignancy along with cytokine-associated toxicity in a clinical trial of anti-CD19 chimeric-antigen-receptor-transduced T cells. Blood 2012;119:2709-20.

49. Lee DW, Gardner R, Porter DL, et al. Current concepts in the diagnosis and management of cytokine release syndrome. Blood 2014;124:188-95.

50. Vignali DA, Collison LW, Workman CJ. How regulatory T cells work. Nat Rev Immunol 2008;8:523-32.

51. Feyler S, von Lilienfeld-Toal M, Jarmin S, et al. CD4(+) CD25(+)FoxP3(+) regulatory $\mathrm{T}$ cells are increased whilst CD3(+)CD4(-)CD8(-)alphabeta TCR(+) double negative T cells are decreased in the peripheral blood of patients with multiple myeloma which correlates with disease burden. Br J Haematol 2009; 144:686-95.

52. Ghiringhelli F, Larmonier N, Schmitt E, et al. CD4+CD25+ regulatory $T$ cells suppress tumor immunity but are sensitive to cyclophosphamide which allows immunotherapy of established tumors to be curative. Eur J

doi: $10.21037 /$ sci-2020-029

Cite this article as: Ding L, Hu Y, Huang H. Novel progresses of chimeric antigen receptor (CAR) $\mathrm{T}$ cell therapy in multiple myeloma. Stem Cell Investig 2021;8:1.
Immunol 2004;34:336-44.

53. Galustian C, Meyer B, Labarthe MC, et al. The anticancer agents lenalidomide and pomalidomide inhibit the proliferation and function of $\mathrm{T}$ regulatory cells. Cancer Immunol. Immunother 2009:58:1033-45.

54. Schlecker E, Stojanovic A, Eisen C, et al. Tumor-infiltrating monocytic myeloid-derived suppressor cells mediate CCR5dependent recruitment of regulatory $\mathrm{T}$ cells favoring tumor growth. J Immunol 2012;189:5602-11.

55. Görgün GT, Whitehill G, Anderson JL, et al. Tumor-promoting immune-suppressive myeloidderived suppressor cells in the multiple myeloma microenvironment in humans. Blood 2013;121:2975-87.

56. Li H, Han Y, Guo Q, et al. Cancer-expanded myeloidderived suppressor cells induce anergy of NK cells through membrane-bound TGF-beta 1. J Immunol 2009;182:240-9.

57. Serafini P, Mgebroff S, Noonan K, et al. Myeloidderived suppressor cells promote cross-tolerance in B-cell lymphoma by expanding regulatory $\mathrm{T}$ cells. Cancer Res 2008;68:5439-49.

58. Vincent J, Mignot G, Chalmin F, et al. 5-Fluorouracil selectively kills tumor-associated myeloid-derived suppressor cells resulting in enhanced T cell-dependent antitumor immunity. Cancer Res 2010;70:3052-61.

59. Hideshima T, Mitsiades C, Tonon G, et al. Understanding multiple myeloma pathogenesis in the bone marrow to identify new therapeutic targets. Nat Rev Cancer 2007;7:585-98.

60. Zhu Z, Singh V, Watkins SK, et al. High-avidity T cells are preferentially tolerized in the tumor microenvironment. Cancer Res 2013;73:595-604.

61. Karyampudi L, Lamichhane P, Scheid AD, et al. Accumulation of memory precursor CD8 T cells in regressing tumors following combination therapy with vaccine and anti-PD-1 antibody. Cancer Res 2014;74:2974-85. 